University of Warwick institutional repository: http://go.warwick.ac.uk/wrap This paper is made available online in accordance with publisher policies. Please scroll down to view the document itself. Please refer to the repository record for this item and our policy information available from the repository home page for further information.

To see the final version of this paper please visit the publisher's website. Access to the published version may require a subscription.

Author(s): S. Dixon, B. Lanyon, and G. Rowlands

Article Title: Coating thickness and elastic modulus measurement using ultrasonic bulk wave resonance

Year of publication: 2006

Link to published version: http://dx.doi.org/10.1063/1.2192144

Publisher statement: None 


\title{
Coating thickness and elastic modulus measurement using ultrasonic bulk wave resonance
}

\author{
S. Dixon, ${ }^{\text {a) }}$ B. Lanyon, and G. Rowlands \\ Department of Physics, University of Warwick, Coventry CV4 7AL, United Kingdom
}

(Received 6 January 2006; accepted 16 February 2006; published online 4 April 2006)

\begin{abstract}
Measurement of the resonant through thickness ultrasonic modes of a homogeneous plate using a fast Fourier transform of the temporal data can be used to calculate plate thickness very accurately. We describe an extension of this principle to two-layer systems, examining a thin coating on a substrate of known properties. The resonant behavior of these systems is predicted and we explain how this approach is used to measure coating thickness and elastic modulus. Noncontact electromagnetic acoustic transducers are used for ultrasonic measurement, as they do not significantly affect the resonant response of the system, unlike alternative contact transducers. (C) 2006 American Institute of Physics. [DOI: 10.1063/1.2192144]
\end{abstract}

While there are a range of techniques designed to measure coating thicknesses, ${ }^{1-3}$ ultrasonic techniques for gauging coating thickness can offer fast and nondestructive measurements. Conventional ultrasonic techniques employ a traveling wave approach where the ultrasonic wavelength is significantly shorter than the coating layer thickness. To directly resolve coatings of the order of $10^{-5} \mathrm{~m}$ thick, frequencies of the order of $100 \mathrm{MHz}$ or greater would be required. Such measurements are impractical in most realistic applications due to difficulties in transduction at these frequencies and correspondingly high levels of attenuation. There have been noncontact laser based surface wave techniques that have successfully measured coating thicknesses for particular substrates. ${ }^{4}$

Recent research has shown that an ultrasonic bulk wave resonance technique can yield highly accurate thickness measurements of uncoated sheets using wavelengths of the order of the sheet thickness. Even though surface roughness can be on the scale of microns; ultrasonic measurements effectively average the thickness over the footprint of the transducer. Thickness of uncoated sheets can be measured to accuracies greater than $10^{-7} \mathrm{~m}$ without contacting the sample. ${ }^{5}$ The noncontact nature is important since direct mechanical contact could significantly alter the characteristic frequencies of the resonant modes.

We recently examined the resonant modes of the twolayer systems formed by a coating and substrate to the system properties. ${ }^{6}$ A general model was derived and examined that relates the frequency of resonant modes to the system properties via the following transendental equation:

$$
\rho_{1} c_{1} \tan \left(\frac{\omega}{c_{1}} d_{1}\right)=-\rho_{2} c_{2} \tan \left(\frac{\omega}{c_{2}} d_{2}\right),
$$

where the subscripts "1" and "2" refer to the substrate and coating, respectively, $\rho$ is density, $c$ is the ultrasonic velocity, $d$ is the layer thickness, and $\omega$ is the angular frequency of the resonant mode. Attenuation in each layer was assumed negligible.

This model was modified to incorporate an element of acoustic attenuation in the coating layer, through the viscos-

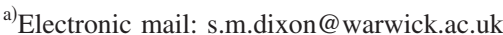

ity of the coating $\eta$, as shown in Eq. (2). Attenuation will not only affect the amplitude of a resonant mode but also has the potential to shift the resonant frequency.

$$
\begin{aligned}
\rho_{1} c_{1} \tan \left(\frac{\omega}{c_{1}} d_{1}\right)= & -\rho_{2} c_{2} \sqrt{1-\frac{i \eta_{2}}{\rho_{2} \omega}} \\
& \times \tan \left(\frac{\omega d_{2}}{c_{2}} \sqrt{1-\frac{i \eta_{2}}{\rho_{2} \omega}}\right) .
\end{aligned}
$$

This development was necessary in order to fully understand the behavior of resonant modes in the system. However, experiments have shown that taking account of attenuation does not significantly affect the measured frequencies of experimentally observable resonant modes. ${ }^{6}$ The simple model of Eq. (1) is a suitable approximation that relates the frequencies of experimentally observable resonant modes to the system properties.

In the particular case that we will use to illustrate the thickness gauging method here, the substrates studied were nominally $220 \mu \mathrm{m}$ thick aluminum sheets and the coatings were approximately $11 \mu \mathrm{m}$ thick epoxy layers.

Since the presence of a thin layer of coating material can significantly affect the resonant mode of the system ${ }^{5}$ the mechanical coupling of an ultrasonic transducer could potentially change the apparent resonant modes of the system as the transducer loads and becomes part of that system. Thus, a noncontact, radially polarized shear wave, wideband electromagnetic acoustic transducer (EMAT) was used for the ultrasonic measurement. EMATs (Refs. 5 and 7-9) operate via the Lorentz mechanism, whereby for ultrasonic generation an eddy current is electromagnetically induced in the skin depth of an electrically conducting sample, which in turn interacts with an orthogonally directed, static magnetic field. This secondary electron current effectively "drags" the crystal lattice with it and in doing so generates an ultrasonic wave. In detection the ultrasonic motion of the sample in a direction orthogonal to the magnetic field generates a current orthogonal to both the direction of particle vibration and static magnetic field. The magnetic field associated with this current is detected by a detection coil, which in our case is the same coil that was used to induce the initial eddy current in the sample. 


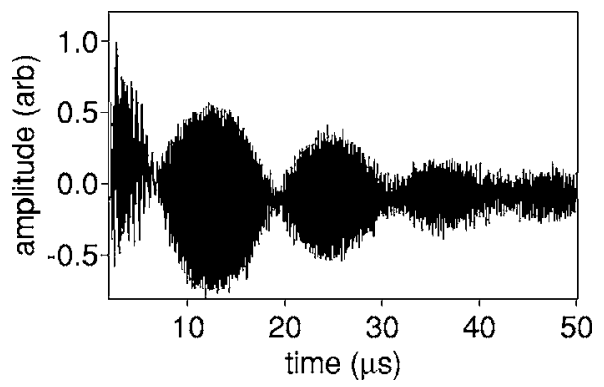

FIG. 1. Time domain wave form (A scan) showing modulation due to interference between the two orthogonal shear wave polarizations for the through thickness shear wave modes of a $0.22 \mathrm{~mm}$ thick aluminum sheet. The inset shows that individual shear wave pulses are not distinguishable.

The temporally sharp shear wave pulse generated by the EMAT has significant frequency content in the range of 0.5-12.0 MHz. Within this range of frequencies, only a discrete set of resonant modes may exist within the two-layer system. For experimentally observable modes, these frequencies can be deduced from Eq. (1) or (2) where a coating is present.

The ultrasonic time domain wave form (or A scan) appears as shown in Fig. 1 for a $220 \mu \mathrm{m}$ thick uncoated aluminum sheet. Note that the ultrasonic echoes appear to overlap and discrete pulses are not visible as is evident on the inset of Fig. 1. Modulation in the A scan is due to interference between the two possible orthogonal shear wave polarizations in the sheet, along and perpendicular to the rolling direction, also known as acoustic birefringence. Minima in the A scan correspond to regions where the shear waves in the orthogonal polarizations are in antiphase. ${ }^{5}$

The magnitude fast Fourier transform (FFT) of this A scan is shown in Fig. 2, where only one peak can be observed due to the bandwidth of the particular EMAT system used. A "zoom in" of this peak together with one from a sheet of the same material with a coating of an approximately $11 \mu \mathrm{m}$ layer of cured epoxy resin is shown in Fig. 3. At this resolution two distinct peaks can be observed for each sample that correspond to the two orthogonal shear wave polarizations in the aluminum sheet. The differences in amplitude between each peak are due to the relative amount of ultrasonic energy steered into each polarization and any slight curvature in the sample which can lead to one polarization being detected and generated more efficiently than the other.

Note that the frequency difference observed in Fig. 3 between the coated and uncoated sheets is significant. By

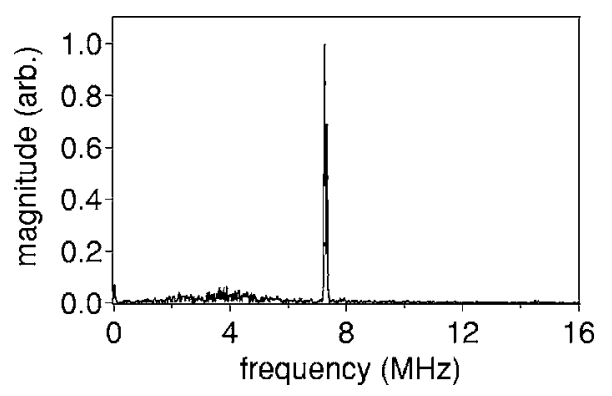

FIG. 2. FFT of the A scan in Fig. 1 showing what is actually a double peak structure centered around $7.3 \mathrm{MHz}$. Only one double peak is visible in this case as the wave form does not contain sufficient ultrasonic signals at the next resonant frequency at approximately $14.6 \mathrm{MHz}$. Downloaded 01 Jul 2009 to 137.205 .202 .8 . Redistribution subject to AIP license or copyright; see http://apl.aip.org/apl/copyright.jsp

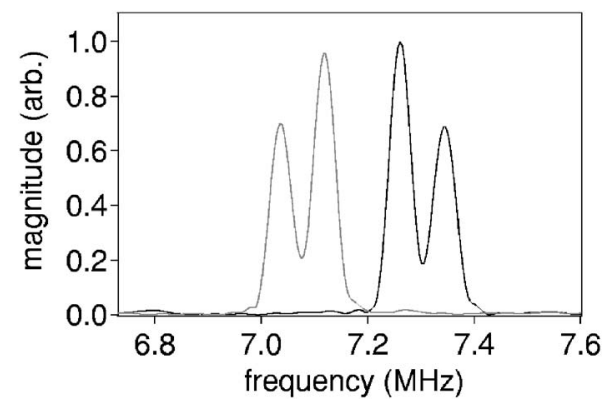

FIG. 3. "Zoomed in" FFTs of the A scans for an uncoated $0.22 \mathrm{~mm}$ thick aluminum sheet (black and on right) and a $0.22 \mathrm{~mm}$ thick aluminum sheet from the same strip with a nominal $11 \mu \mathrm{m}$ thick polymer coating (gray and on left). Note that the double peak structure is due to acoustic birefringence in the aluminum.

using the resonant modal frequencies of the coated sample results with Eq. (1) we can calculate the thickness of the coating for given values of density and shear wave velocity. Note that this must be done numerically since Eq. (1) is a transcendental equation.

Using the manufacturer's data, coating thickness was nominally quoted as $11 \pm 1 \mu \mathrm{m}$, determined by weighing the sample. Using typical values of the shear wave velocity in the epoxy coating of $1100 \mathrm{~m} \mathrm{~s}^{-1}( \pm 10 \%)$ and a density of $1250 \mathrm{~kg} \mathrm{~m}^{-3}( \pm 5 \%)$ yields a calculated thickness measurement of $10.5 \pm 1 \mu \mathrm{m}$. The measured and expected thicknesses agree within experimental error. The limiting factor on the accuracy of ultrasonic measurement is the precision to which the density and shear wave velocity are known. Because of the form of Eq. (1) the calculation is most sensitive to uncertainties in wave velocity. In this system a $10 \%$ uncertainty in velocity leads to approximately a $10 \%$ uncertainty in the calculated thickness while a $10 \%$ uncertainty in the density leads to only a $1 \%$ uncertainty in the calculated thickness.

With a wider bandwidth EMAT system or a thicker substrate, we are able to measure the position of more than one peak. Dividing Eq. (1), for an experimentally measured mode $\omega_{n}$ by Eq. (1) for another known modes $\omega_{m}$, yields

$$
\frac{\tan \left(\omega_{n} d / c_{1}\right)}{\tan \left(\omega_{m} d / c_{1}\right)}=\frac{c_{2} \tan \left(\omega_{n} h / c_{2}\right)}{c_{2}^{\prime} \tan \left(\omega_{m} h / c_{2}^{\prime}\right)}
$$

where $c_{2}$ is the polymer phase velocity at frequency $\omega_{n}$ and $c_{2}{ }^{\prime}$ is the polymer phase velocity at frequency $\omega_{m}$. If we neglect viscoelastic behavior of the coating (change in ultrasonic velocity with frequency) then Eq. (3) can be solved for the ratio of the polymer properties $h / c_{2}$. With knowledge of the density of the coating, which is often a well defined parameter, Eq. (1) can then be used to determine both the polymer layer thickness and phase velocity.

Many materials would have an insignificant variation in elastic modulus over the frequency ranges that we describe here. Neglecting viscoelastic behavior of polymer coatings typically results in an error of less than a few percent in the calculated coating properties. The error translates approximately linearly with the error in velocity.

In conclusion we present a generic technique for determining ultrasonically, coating thickness, having demonstrated the method in a system where it would be very diffi- 
cult to measure by conventional ultrasonic means. Where viscoelasticity can be ignored then, with knowledge of the coating density and more than one resonant mode, both the shear elastic modulus and thickness of the coating can be determined. In conventional contacting ultrasonic measurements, thin layers of couplant are often used and one would not be able to discriminate between the effects due to a thin couplant layer or a coating layer. The use of noncontact ultrasonics offers a significant advantage for this measurement problem.
${ }^{1}$ R. Wang and P. L. Wong, Tribol. Int. 30, 801 (1997).

${ }^{2}$ S. K. Lau, D. P. Almond, and P. M. Patel, J. Phys. D 24, 428 (1991).

${ }^{3}$ A. I. Lavrentyev and S. I. Rokhlin, Ultrasonics 39, 211 (2001).

${ }^{4}$ D. C. Hurley, V. K. Tewary, and A. J. Richards, Meas. Sci. Technol. 12, 1486 (2001)

${ }^{5}$ S. Dixon, C. Edwards, and S. B. Palmer, Ultrasonics 39, 445 (2001).

${ }^{6}$ S. Dixon, B. Lanyon, and G. Rowlands, J. Phys. D 39, 506 (2006).

${ }^{7}$ H. M. Frost, in Physical Acoustics XIV, edited by W. P. Mason and R. N. Thurston (Academic, New York, 1979), pp. 179-275.

${ }^{8}$ E. R. Dobbs, in Physical Acoustics X, edited by W. P. Mason and R. N. Thurston (Academic, New York, 1973), pp. 127-189.

${ }^{9}$ K. Kawashima, J. Acoust. Soc. Am. 60, 1089 (1976). 